\title{
Volume and Surface Resistivity Measurement of Insulating Materials Using Guard-Ring Terminal Electrodes
}

\author{
Heon-Gyeong Lee ${ }^{\circledR}$ and Jin-Gyu Kim * $*$ \\ High Voltage \& Plasma Application Lab. (HVPAL), Department of Electrical Engineering, \\ KyungPook National University, Daehakro, Bukgu, Daegu 41566, Korea; hglee0211@knu.ac.kr \\ * Correspondence: kjg@knu.ac.kr; Tel.: +82-053-950-7226
}

Received: 5 May 2020; Accepted: 31 May 2020; Published: 1 June 2020

check for updates

\begin{abstract}
Recently, eco-friendly energy conversion policies have been being promoted through de-nuclearization and de-coal. For this purpose, a super grid should be built to optimize sustainable renewable energy resources such as solar and wind power. Accordingly, considering the various problems such as technology and cost, a system for efficient energy transmission is required. Hence, research is being actively conducted to apply it, owing to the development of the high voltage direct current (HVDC) system. Among HVDC systems, the cable system is extremely important, in addition to the measurement of the dielectric breakdown strength, space charge, and volume resistivity of insulating materials. The existing resistivity measurement method measures both the volume and surface resistivity using a three-terminal electrode that is used in the international standards of American Society for Testing and Materials (ASTM) D 257 and International Electrotechnical Commission (IEC) 60093. However, the circuit configuration differs depending on the measurement of the volume and surface resistivity; moreover, when a DC voltage is applied to the insulator, a charging current flows and there are multiple samples to be measured, which takes a considerable amount of time. Therefore, in this study, we proposed a new type of resistivity measurement system that is based on the existing three-terminal electrode system. Furthermore, we produced a system capable of simultaneously measuring the volume and surface resistivity. Finally, using this system, we compared and analyzed the volume and surface resistivity of five insulating materials.
\end{abstract}

Keywords: volume resistivity; surface resistivity; leakage current

\section{Introduction}

Both developing and developed countries are facing a transition period for electrification. Because of the increase in power demand in city centers in large cities, several studies have focused on efficiently transmitting electricity between cities, countries, and continents. Moreover, over the past few years, an eco-friendly energy conversion policy has been promoted via de-nuclearization and de-coal. For this purpose, a new super grid is required to optimize sustainable renewable energy resources such as solar and wind power. European and G8 leaders have announced that, by 2050, they will cut their countries' greenhouse gas emissions by $80 \%$. Instead of improving the system of an existing high voltage alternating current (HVAC) grid, they intend to use the HVDC grid to interconnect EU countries. Furthermore, Northeast Asia is pushing for the efficient transmission of power energy and power production via nuclear power plants and renewable energy [1-3].

Such transmission technology should be selected while considering both social and environmental impacts and limitations such as technology and cost. Accordingly, the HVDC system is considered a system for efficient energy transmission. The existing HVAC system has been a pivotal power 
transmission technology for $>100$ years; however, the HVDC transmission system's importance has emerged because of the increase in power transmission capacity and the liberalization of the power industry. Generally, the HVDC has a higher allowable current of the conductor than the HVAC; therefore, it can transmit more power, which can reduce the equipment cost of pylons and wiring. Moreover, because the HVAC system suffers from high loss because of the limitation of reactive power during long-distance transmission, studies on the application of a HVDC transmission system capable of relatively extended loss and large-capacity transmission have been actively conducted. Because the HVDC system is operated in a DC environment rather than an existing AC environment, a new insulation design technology is required. Moreover, additional research is required to develop power devices to apply this system and improve insulation performance [1,4-7]. For an insulating material of an electric power device exposed in an external environment, deterioration occurs where the chemical and mechanical properties of the material change because of environmental factors, thereby deteriorating the insulating property. Note that it is extremely important to understand the reliability of the insulating material because the deteriorated insulating material loses insulating properties and the leakage current flows and eventually breaks the insulation, which can lead to serious accidents [8].

The HVDC system can be divided into operation, conversion, and cable systems; moreover, it is the most important element of the cable system. The most important properties of the insulation material for cables are insulation breakdown strength, space charge, and volume resistivity. Generally, the AC cable has a capacitive electric field distribution determined by the dielectric constant; however, the DC cable has a resistive electric field distribution determined by the resistivity. Moreover, regardless of changes in the shape, size, and measurement position, because the resistivity measurement has the inherent electrical properties of the insulating material, a reliable measurement is required to apply the insulating material to power equipments. Measuring the volume and surface resistivity of these high-resistivity dielectrics is the most difficult task. The method of measuring the volume and surface resistivity of the insulating material is described in detail in the international standard of IEC 60093 and ASTM D 257 approved in 1980 by the International Electrotechnical Commission; furthermore, both the volume and surface resistivity are measured using a three-terminal electrode $[9,10]$. The resistivity measurement results are greatly influenced by factors such as the applied voltage, electrode materials and shape, temperature, relative humidity, and surface and space charges stored in the sample before measurement, causing errors. Moreover, since the circuit diagram is different depending on the measurement of the volume and surface resistivity, a simultaneous measurement is impossible; therefore, there are disadvantages in individual measurements. Furthermore, for the DC electric field, when an electric field is applied to the dielectric, an absorption phenomenon occurs because of polarization; therefore, it must be measured after a sufficient time for the instantaneous charging current to escape because of the charging current [11-14]. For a voltage applied for measuring the resistivity, it is measured at a voltage as low as 500-1000 V; if the thickness of the specimen is difficult to process, it cannot be measured.

In this study, based on the existing three-terminal electrode system for measuring the volume and surface resistivity, we present a new type of resistivity measurement system. Furthermore, a system capable of simultaneously measuring the volume and surface resistivity is developed. Moreover, we can see the change in resistivity depending on the voltage magnitude. Finally, the volumes and surface resistivities can be compared and analyzed using four insulating materials: polyethylene (PE), polypropylene (PP), polytetrafluoroethylene (PTFE), and polyvinylchloride (PVC).

\section{Experimental Section}

\subsection{Specimens}

In this experiment, we used four types of polymers (PE, PP, PTFE, and PVC) as samples that were used as general insulating materials. The sample sizes suggested in ASTM D 257 and IEC 60093 have 
diameters of 50-100 $\mathrm{mm}$ and thicknesses of $1 \mathrm{~mm}$. However, in this experiment, we used a diameter of $100 \mathrm{~mm}$.

\subsection{Theoretical Background}

Polymer materials are often used in power devices because of their excellent electrical insulation properties. Recently, research channeled towards improving electrical insulation properties has been actively conducted by mixing nanofillers such as $\mathrm{h}-\mathrm{BN}$ and $\mathrm{MgO}$ with insulating materials. Both polarization and charge injection phenomena occur when an electric field is formed in the polymer. Polarization is a phenomenon in which dipoles, which are electric charges existing in an insulator, are arranged according to the direction of an electric field applied from the outside, while charge injection is a phenomenon in which electric charges are injected from a conductor such as an electrode into an insulator when a high electric field is applied. When these phenomena accumulate in the polymer over a long period, they have considerable influence on the dielectric strength. In particular, a treeing phenomenon occurs in cable insulation, causing considerable problems [15-18].

The electric field exhibits different characteristics because of the movement of positive and negative charges, and Equation (1) gives the governing equation for the potential distribution that forms the electric field using the definition of the electric field of the current continuous equation, the Gaussian law, and Ohm's law:

$$
\sigma \nabla^{2} V+\frac{\partial}{\partial t} \varepsilon \nabla^{2} V=0
$$

The governing equation of Equation (1) can be expressed as Equation (2) by rearranging the conductivity and dielectric constant as differential equations over time:

$$
\left(\sigma+\varepsilon \frac{\partial}{\partial t}\right) \cdot\left(\frac{\partial^{2} V}{\partial x^{2}}+\frac{\partial^{2} V}{\partial y^{2}}+\frac{\partial^{2} V}{\partial z^{2}}\right)=0
$$

In the DC electric field distribution, there is no differential term with respect to time; therefore, the coefficient of the dielectric constant becomes 0 . Hence, the conductivity term dominates, yielding an equation for obtaining the DC electric field distribution:

$$
\sigma \cdot\left(\frac{\partial^{2} V}{\partial x^{2}}+\frac{\partial^{2} V}{\partial y^{2}}+\frac{\partial^{2} V}{\partial z^{2}}\right)=0
$$

Furthermore, for the DC insulation, because the voltage is always applied with the same polarity, the influence of the space charge injected into the insulator is considerable. If it is assumed that a negative voltage is applied, the effective insulation thickness decreases because of the expansion of the electric field region due to the continuous accumulation of electrons. At this time, when the opposite polarity (positive polarity) flows through the conductor, a very large electric field is formed, and a local insulation breakdown phenomenon occurs. Therefore, to measure the dielectric breakdown strength and conductivity, it is very important to measure the resistivity [19].

\subsubsection{Resistivity Measurement}

Because the conductivity of the solid dielectric is the reciprocal of the resistivity, the specimen to be measured is placed between the conductive electrodes, and then the resistance is measured. The resistance value is then calculated by measuring the current flowing through the sample at the voltage applied to the electrode. The value is affected by the dimensions of the electrode and the specimen. Note that it can be measured through the three-terminal electrode measurement method suggested by the international standard IEC 60093 (methods of testing for the volume resistivity and conductance of solid electrical insulating materials) and ASTM D 257 (DC resistance or conductance of insulating materials). 
Figure 1a shows the abovementioned three-terminal electrode structure. It comprises three electrodes (top, bottom, and guard electrodes). Figure $1 \mathrm{~b}$ is a plane figure of each electrode and should be configured using Equation (4).

$$
D_{0}=\frac{D_{1}+D_{2}}{2}
$$

where $D_{0}$ is the product of the distance from the origin to the midpoint of $g, D_{1}$ is the diameter of the bottom electrode, $D_{2}$ is the diameter of the bottom electrode $+\mathrm{g}$ (spacing between the guard and bottom electrode), and $D_{3}$ is the diameter of the top electrode.

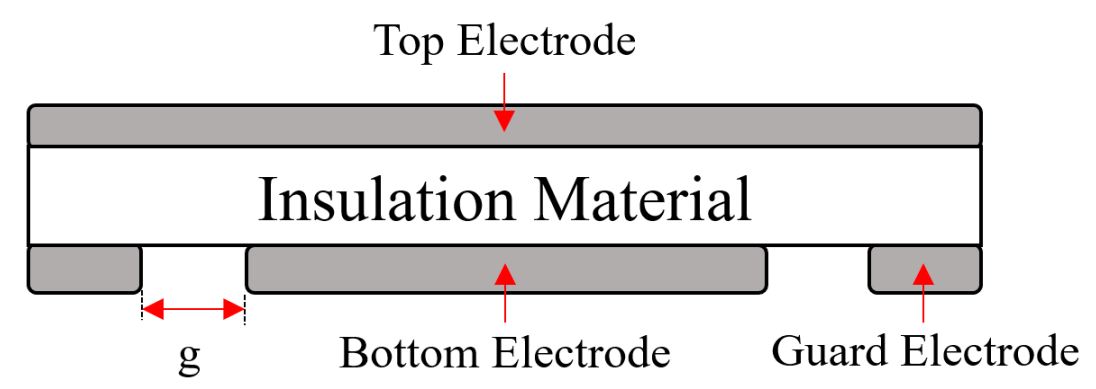

(a)

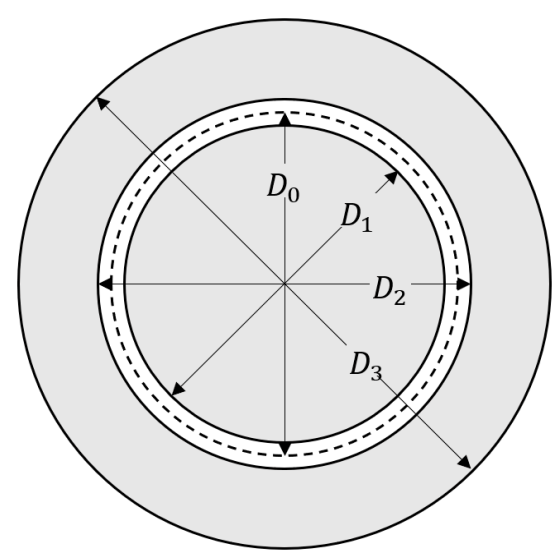

(b)

Figure 1. Specimen for measuring the volume and surface resistance: (a) electrode structure; (b) flat specimen electrode.

When measuring the resistivity, a three-terminal electrode is installed on an insulator. When a DC high voltage is applied, the generated leakage current is measured and converted into a resistivity. Figure 2 shows the circuit diagram for measuring the volume resistivity: $(+)$ is applied to the top electrode, and (-) is applied to the bottom and guard electrodes. The current flowing from the top electrode to the guard electrode is designed to reduce the error in the volumetric resistance measurement by flowing directly to the ground.

Through the modeling of the circuit diagram for measuring the volume resistivity, the flow of the volume leakage current can be seen (Figure 3). The leakage current flows through the volume of the insulating material at the top electrode, and the measured leakage current is calculated as the volume resistivity using Equations (5) and (6). Depending on the leakage current, the measured resistivity can be measured to about G-P $\Omega[20,21]$.

$$
\begin{gathered}
R_{v}=\rho_{v} \frac{l}{A}=\frac{V_{a}}{I_{L}} \\
\rho_{v}=R_{v} \cdot \frac{A}{d}=R_{v} \cdot \frac{\pi r^{2}}{d}
\end{gathered}
$$


where $R_{v}$ is the volume resistance, $\rho_{v}$ is the volume resistivity, $I_{L}$ is the leakage current, $V_{a}$ is the applied voltage, $d$ is the sample thickness, and $A$ is the main electrode area.

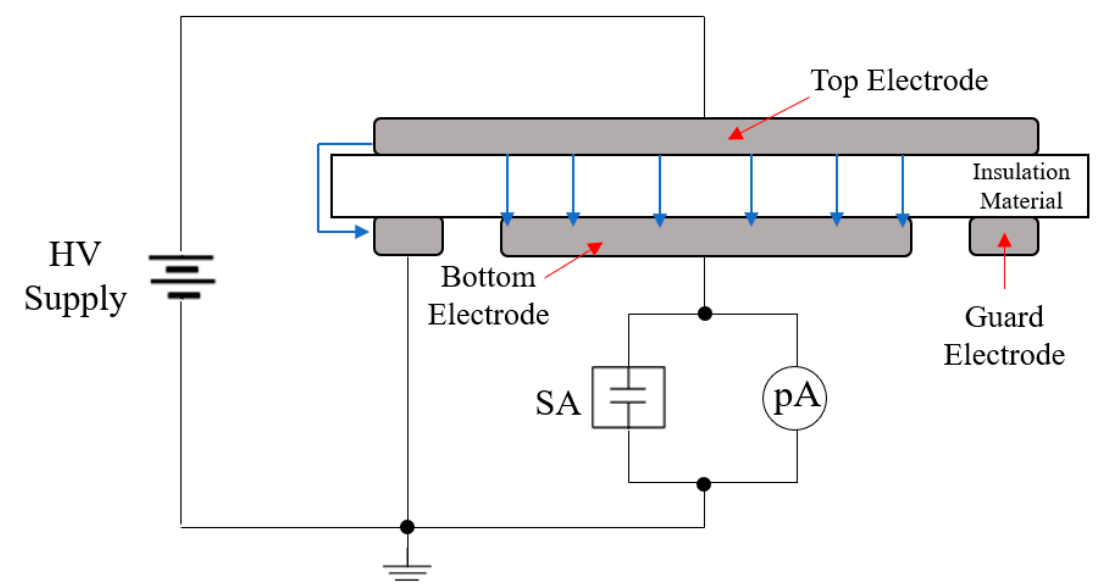

Figure 2. Circuit diagram for measuring the volume resistivity.

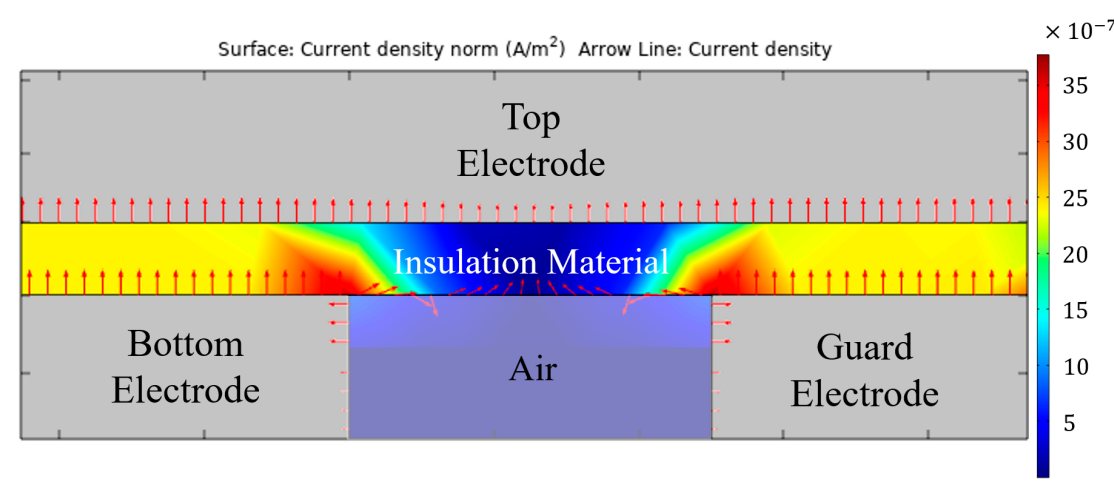

Figure 3. Volume leakage current density and flow.

Figure 4 is the circuit diagram for measuring the surface resistivity. Unlike the circuit for measuring the volume resistivity, $(+)$ is applied to the guard electrode and $(-)$ is applied to the two flat (top and bottom) electrodes. Then, the surface leakage current flowing between the guard electrode and the bottom electrodes can be measured to obtain the resistivity using Equation (7). Because the leakage current flowing from the guard electrode to the top electrode flows to the ground, it does not affect the surface leakage current measurement $[22,23]$. Figure 5 is a simulation of the circuit diagram for measuring the surface resistivity. Since the guard electrode is $(+)$ and the lower and upper electrodes are $(-)$, it can be seen that the current flows differently from the volume resistivity. It can be seen that a current flows from the guard electrode to the lower electrode, and there is a constant current over the upper part.

$$
\rho_{s}=R_{s} \frac{2 \pi}{\ln \left(D_{2} / D_{1}\right)}
$$

where $R_{s}$ is the surface resistance, $\rho_{s}$ is the surface resistivity, and $D_{1}$ and $D_{2}$ are the diameters of the inner and outer electrodes, respectively.

As shown in Figures 2 and 4, the circuits are configured differently, according to the volume and surface resistivity measurements. Since the leakage current flows are different, a simultaneous measurement is impossible and individual measurements must be made. 


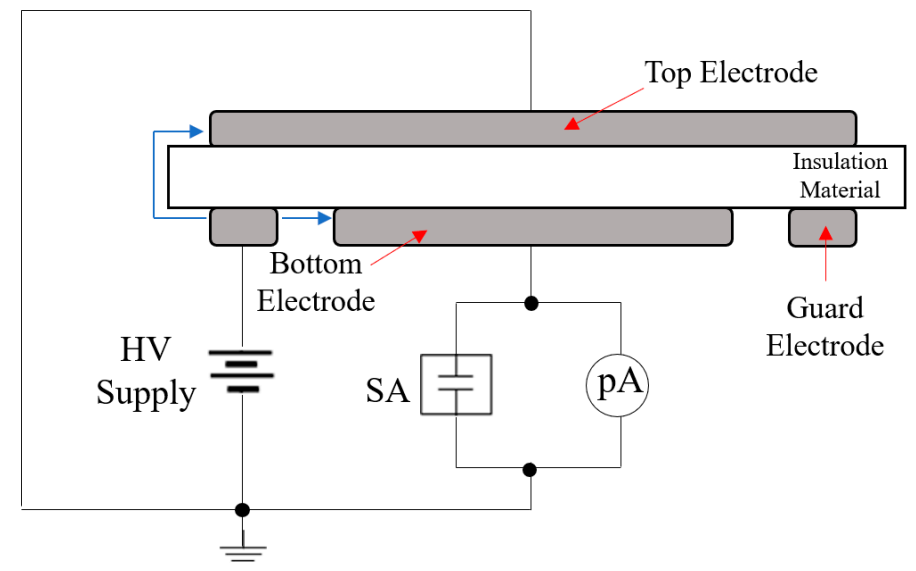

Figure 4. Circuit diagram for measuring the surface resistivity.

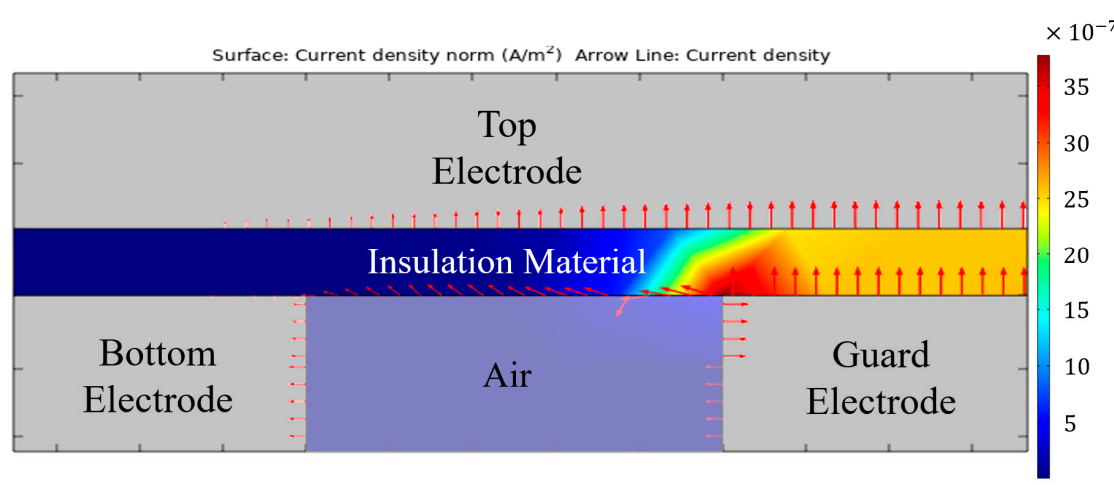

Figure 5. Surface leakage current density and flow.

\subsubsection{Charging Current}

For general insulating materials, when an electric field is applied to the dielectric, it is one dielectric, and various other types of polarization occur. Because of this polarization phenomenon, immediately after the DC voltage of the insulating material is applied, the instantaneous charging current $I_{\mathcal{c}}$ flows and tends to decrease as the application time increases. This polarization phenomenon is called the absorption phenomenon of the dielectric, and the decreasing current is called the absorption current $I_{a}$. For an ideal insulator, the current is almost zero; however, in a real environment, a constant microcurrent is observed, even after a sufficient time. This constant current is called the leakage current $I_{l}$, and Figure 6 is the current flowing through the insulator when the DC voltage is applied.

- $\quad I_{\mathcal{C}}$ (Instantaneous charging current): It is the current part that rapidly rises along with the voltage, and it refers to the current component generated by the electrostatic capacity and electron and atomic polarization formed in the insulator.

- $\quad I_{a}$ (Absorption current): It is the current part that continuously flows and gradually decreases in the charging current, and it refers to a current component based on a relatively gentle electric polarization (such as orientation and interface polarizations).

- $I_{l}$ (Leakage current): It means the current part reaching a steady state where the current is maintained at a constant value. It is very difficult to calculate the pure leakage current in a short time.

Both the volume and surface resistivity can be calculated by measuring the leakage current. In general, after applying a particular voltage, select a certain time period (5, 10, and $30 \mathrm{~min}$, etc.), wait for the instantaneous charging current to drain, and then decrease the current. The current (leakage current) at this time becomes constant. Note that there is a problem that involves taking a long time when many samples have to be measured [24,25]. 


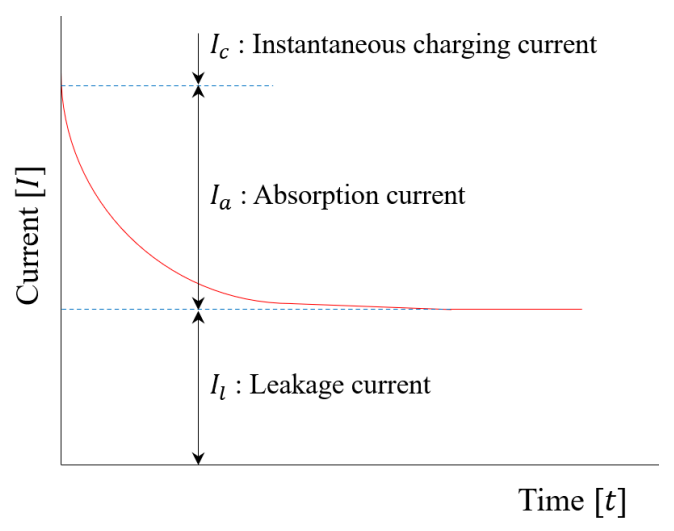

Figure 6. Graph of the current when the DC voltage is applied to the dielectric.

\subsubsection{Guard-Ring Terminal Electrode Type Resistivity Measurement System}

A new guard-ring type resistivity measurement system is proposed to improve the difference between the volume and surface resistivity measurement circuit configurations and the long-time measurement time based on the charging current in the three-terminal electrode resistivity measurement methods of IEC 60093 and ASTM D 257. The shape of the guard-ring electrode is modified from the basic frame of the existing three-terminal electrode, and the top and bottom electrodes are identically configured. For the guard-ring electrode, it has a shape attached to the side and bottom of the insulator; moreover, it is configured as shown in Figure 7. For there to be a perfect contact of the electrode to the edge of the material, it must have a tight tolerance. To this end, the ring electrode on the side has a thickness of $0.5 \mathrm{~mm}$, so that it is easy to bend. Using this, it was adhered to the material and used as a single electrode by attaching it to the bottom ring electrode and to the outside using conductive tape.

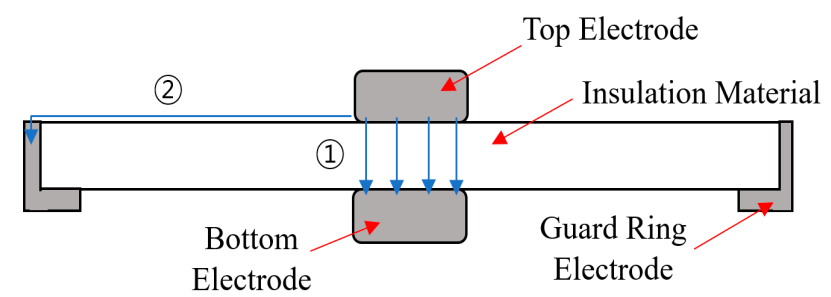

Figure 7. Guard-ring terminal electrode structure for measuring insulator resistivity.

The flow of the volume and surface leakage current when the voltage is applied is as shown in (1) and (2) in Figure 7. For each leakage current measurement and the flow of the leakage current, the voltage application method is as follows.

- (1) When the voltage for measuring the volume resistivity is applied $(+)$ to the top electrode and $(-)$ to the bottom and guard-ring electrodes, the internal leakage current of the insulator flows through the top and bottom electrodes.

- (2) The voltage application method of the surface resistivity measurement is applied in the same way as the volume resistivity measurement. In the surface leakage current of the insulator, a leakage current generated from the top electrode flows along the surface of the insulator to the guard-ring electrode on the side surface.

Table 1 shows the parameters of the electrode and the insulating material used in this study. For the electrode, stainless steel, which is relatively easy to process and has excellent corrosion resistance, was used because the material and size have a large influence on the measurement results. After modeling was performed through the parameters in Table 1, power was connected to each electrode. The current density distribution and leakage current flow generated at this time were simulated. 
Table 1. Parameters of electrodes and insulating materials.

\begin{tabular}{cc}
\hline Item & \\
\hline Top electrode & diameter: $60 \mathrm{~mm} \Phi$ \\
Bottom electrode & diameter: $60 \mathrm{~mm} \Phi$ \\
& inner diameter: $90 \mathrm{~mm} \Phi$ \\
Guard-ring electrode & outer diameter: $100 \mathrm{~mm} \Phi$ \\
& highest: $1 \mathrm{~mm}$ \\
Insulation material & diameter: $100 \mathrm{~mm} \Phi$ \\
& thickness: $1 \mathrm{~mm}$ \\
\hline
\end{tabular}

Figure 8 shows the simulation results. As shown in Figure 8, the internal leakage current flows from the top electrode to the bottom electrode, and the surface leakage current flows from the top electrode to the guard-ring electrode. Moreover, the leakage current flows from the bottom electrode to the guard-ring electrode, which indicates that the electric current flows as a result of the potential difference attributed to the formation of an electric field because the insulator is a dielectric material. However, because it has a very small value, it is considered not to cause a large error in measurement.

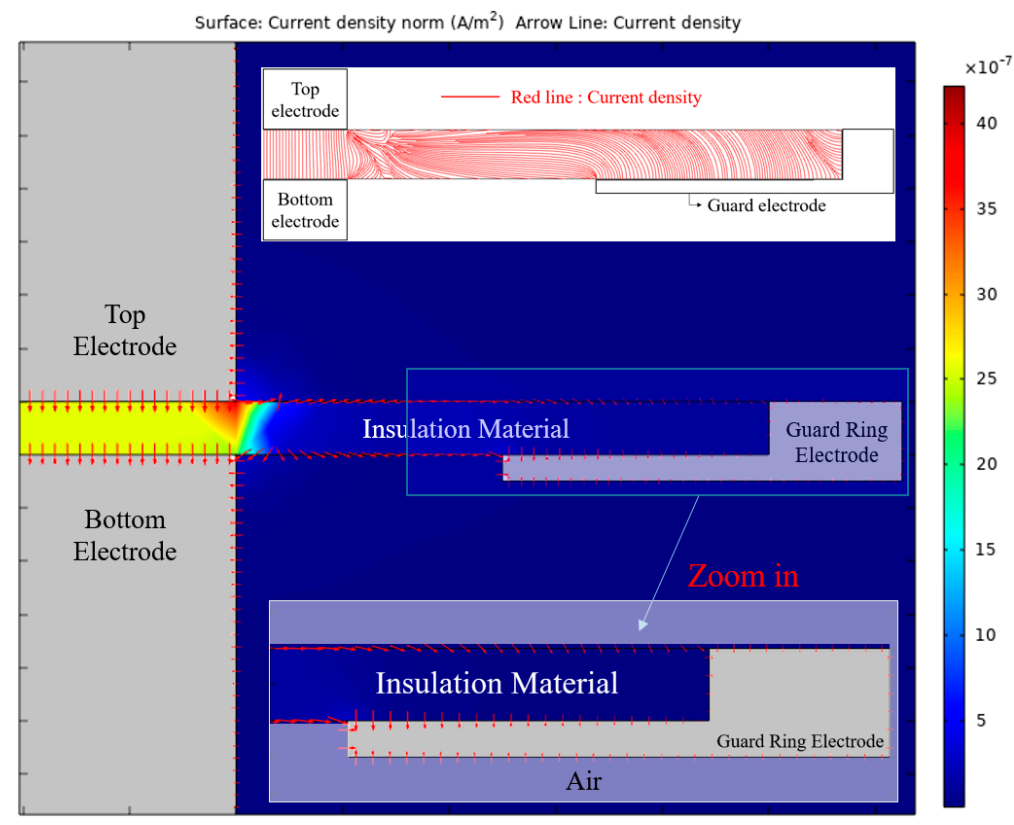

Figure 8. Current density simulation of the guard-ring terminal electrode structure for measuring the insulator resistivity.

Figure 9 shows the circuit diagram of the guard-ring terminal electrode structure for measuring the volume and surface resistivity. The volume and surface leakage current can be simultaneously measured. At this time, if a potential difference occurs in two measurement parts or if, due to a failure, a fault current does not fall to the ground and flows back to the measuring device, which affects the measurement, a large error is introduced in the measurement result. Therefore, to prevent reverse currents, the diodes $D_{1}$ and $D_{2}$ were installed after the measurement devices were installed on each electrode to increase the reliability of the measured values.

To measure the surface resistivity through the existing three-terminal electrode, because the size of the bottom and guard-ring electrodes varies depending on the size of the top electrode, there is a relatively short interval between the electrodes; therefore, there is a disadvantage in the fact that the applied voltage for measuring the resistivity is low. This is because a corona discharge may occur between electrodes as the applied voltage increases because of a short electrode interval; furthermore, the corona current generated at this time may be measured and may cause an error. 
However, because the electrode structure that is proposed is a wide electrode spacing for the surface resistivity measurement, it is possible to measure resistivity even at a high voltage. Moreover, because there is a difference in the resistivity value according to the voltage magnitude, this measurement system can measure the change in the resistivity for each voltage magnitude.

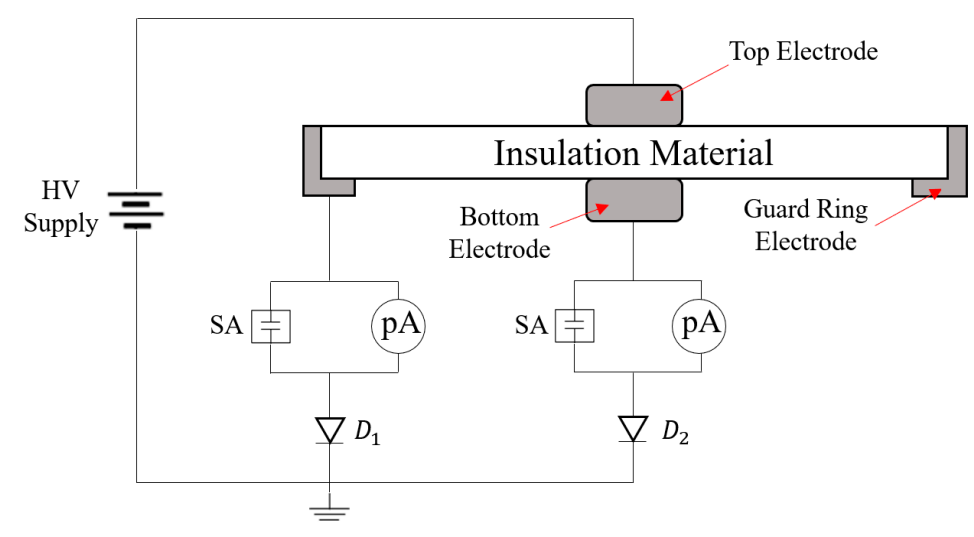

Figure 9. Circuit diagram of the guard-ring terminal electrode structure for measuring the insulator resistivity.

\subsection{Experimental Methods}

Figure 9 shows the schematic of the experiment to measure the volume and surface resistivity of the insulating material. DC voltage is applied to the top electrode, and the ground is connected to the bottom and guard-ring electrodes. To avoid exposure to the external environment, the experimental apparatus was installed in an insulated chamber with temperature and humidity control. Note that the experiment was carried out in air $\left(21 \pm 2{ }^{\circ} \mathrm{C} 32 \pm 2 \% \mathrm{RH}\right)$ in an experimental chamber. The high voltage DC power source (0-30 kV, $20 \mathrm{~mA}$, Spellman, Suzhou, China) is used, and a Keithley picoammeter, model 485 is used to measure the generated leakage current. The applied voltage increased by $2 \mathrm{kV}$, and the leakage current was measured after waiting for a sufficient time period to reduce the influence on the charging current after applying the voltage.

\section{Results and Discussion}

Figure 10 shows a typical time chart of the volume and surface leakage current of PP at an applied voltage of $500 \mathrm{~V}$. Because of the polarization, the instantaneous charging current flows from the start of the DC voltage application, and the absorption current attenuates over a certain time. The leakage current $\left(I_{a}\right)$ flows stably and continuously after the reduction of the sufficient absorption current has started. From the results of the experiment, it is judged that a sufficient reduction started at about $500 \mathrm{~s}$.

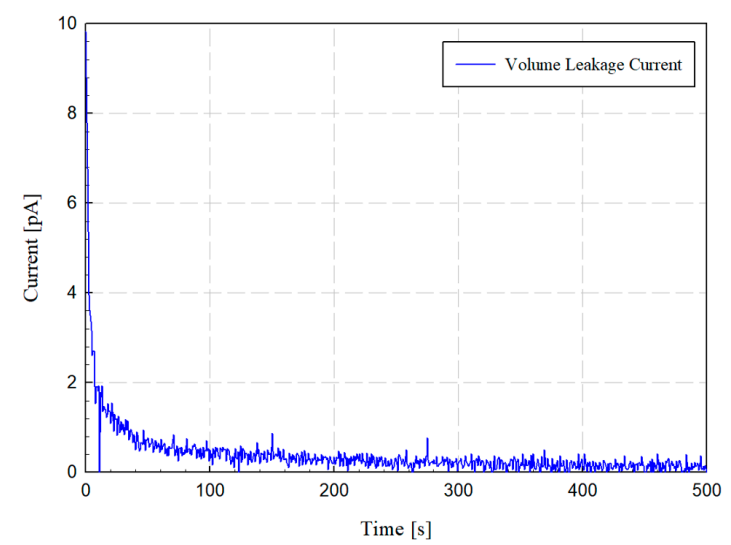

(a)

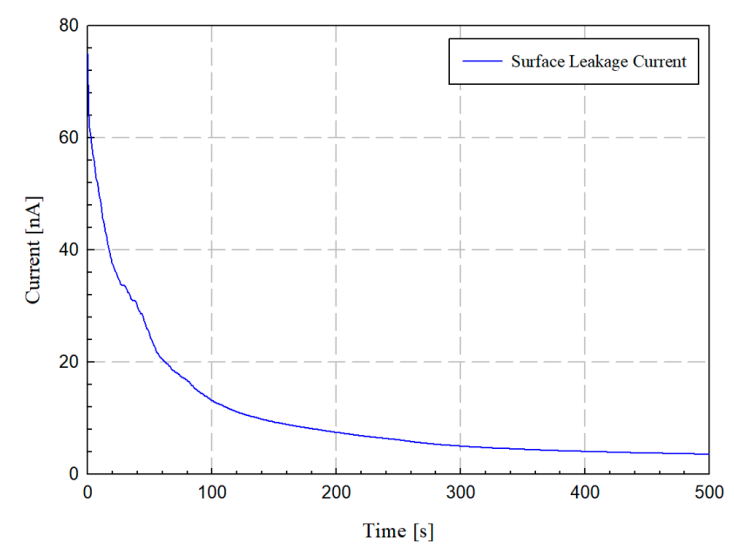

(b)

Figure 10. Volume and surface leakage current time characteristics obtained for the PP (a) volume leakage current; (b) surface leakage current. 
Figure 11 shows the volume and surface leakage current measured through the guard-ring terminal electrode structure proposed in this work. For the volume leakage current, according to the insulating material, the applied voltage showed a rapid increase in the current at around $20 \mathrm{kV}$, and the surface leakage current increased at about $15 \mathrm{kV}$. Note that the corona discharge occurred as the surface insulation between the top electrode and the guard-ring electrode was destroyed with the increase in voltage; furthermore, the current rapidly increased because of the corona current. Therefore, when the voltage increased to some extent, the measurement of the resistivity was inaccurate at the point where the current rapidly increased because of corona discharge. If the sizes of the top and bottom electrodes are changed, the generation of leakage current and the timing of corona discharge are different. As a result of the experiment, it is understood that PVC has the largest volume and surface leakage current, which indicates that both the surface insulation and volume are weak.

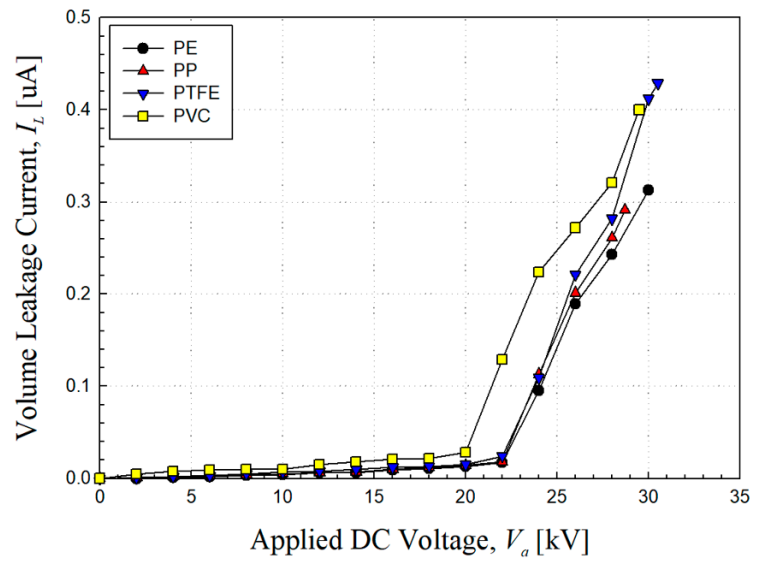

(a)

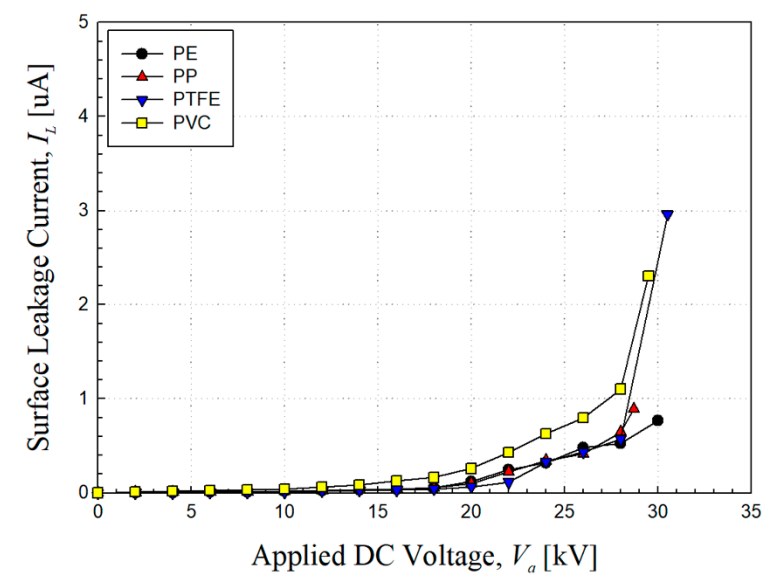

(b)

Figure 11. Volume and surface leakage current characteristics for each insulating material according to the applied DC voltage: (a) volume leakage current; (b) surface leakage current.

The resistivity measurement must be completely discharged before measuring the current because the residual charge of the insulator can considerably affect the results. Figure 12 shows the resistivity of each insulating material based on the volume and surface leakage current measured above. When measuring the resistivity, the volume resistivity tends to decrease rapidly at $20 \mathrm{kV}$, and the surface resistivity decreases at $15 \mathrm{kV}$ because it is based on the leakage current. Because of the experiment, Table 2 shows the volume resistivity $(20 \mathrm{kV})$ and the surface resistivity $(18 \mathrm{kV})$ of each insulating material. For the volume resistivity, PE is the largest with $233.46 \mathrm{~T} \Omega \cdot \mathrm{m}$, and PVC is the smallest with 124.16 T $\Omega \cdot \mathrm{m}$. Meanwhile, for the surface resistivity, PTFE is the largest with $1.66 \mathrm{~T} \Omega \cdot \mathrm{m}$, and PVC is the smallest with $0.44 \mathrm{~T} \Omega \cdot \mathrm{m}$. The volume resistivity was measured in the order of PE $>$ PP $>$ PTFE $>$ PVC, and the surface resistivity was measured in the order of PTFE $>$ PP $>$ PE $>$ PVC. Furthermore, the volume resistivity of PVC increased from 2 to $10 \mathrm{kV}$ and then tended to decrease. The resistivity then increased and decreased because the leakage current did not flow as much as the voltage increased [26].

Table 2. Volume and surface resistivity of insulating materials.

\begin{tabular}{ccccc}
\hline Materials & $\begin{array}{c}\text { Volume Resistivity } \\
\rho_{v}(20 \mathrm{kV})\end{array}$ & $\begin{array}{c}\rho_{v} \\
\text { Standard Devation }\end{array}$ & $\begin{array}{c}\text { Surface Resistivity } \\
\rho_{\boldsymbol{s}}(18 \mathbf{k V})\end{array}$ & $\begin{array}{c}\rho_{s} \\
\text { Standard Devation }\end{array}$ \\
\hline $\mathrm{PE}$ & $233.46 \mathrm{~T} \Omega \cdot \mathrm{m}$ & 11.87 & $1.32 \mathrm{~T} \Omega \cdot \mathrm{m}$ & 0.094 \\
$\mathrm{PP}$ & $227.62 \mathrm{~T} \Omega \cdot \mathrm{m}$ & 9.42 & $1.40 \mathrm{~T} \Omega \cdot \mathrm{m}$ & 0.063 \\
$\mathrm{PTFE}$ & $210.11 \mathrm{~T} \Omega \cdot \mathrm{m}$ & 10.38 & $1.66 \mathrm{~T} \Omega \cdot \mathrm{m}$ & 0.076 \\
$\mathrm{PVC}$ & $124.16 \mathrm{~T} \Omega \cdot \mathrm{m}$ & 10.86 & $0.44 \mathrm{~T} \Omega \cdot \mathrm{m}$ & 0.088 \\
\hline
\end{tabular}




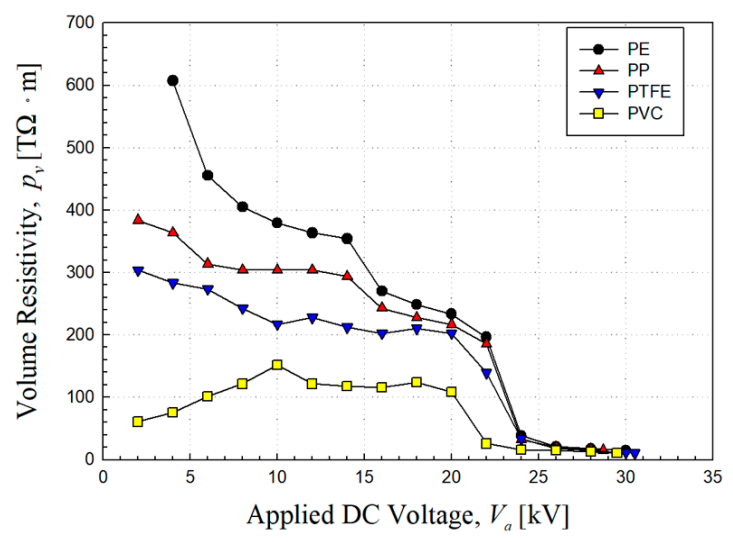

(a)

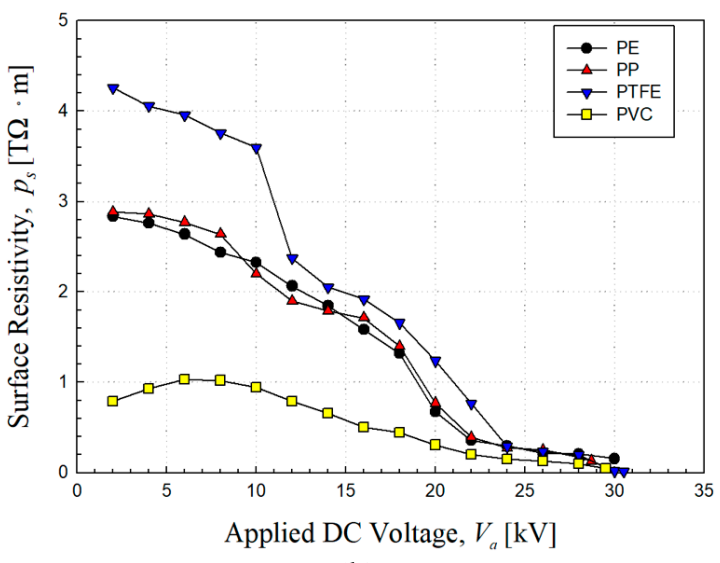

(b)

Figure 12. Volume and surface leakage resistivity characteristics for each insulating material according to the applied DC voltage: (a) volume resistivity; (b) surface resistivity.

\section{Conclusions}

Due to the development of the HVDC system, research to apply it has been actively conducted, and among the HVDC systems, the cable system is the most important element. The measurement of the dielectric breaking strength, space charge, and volume resistance of materials used for cable insulation is of great importance, and the existing method of measuring resistance is performed through three terminal electrodes used in the International Standard Specification of ASTM D 257 and IEC 60093. In the case of the existing three-terminal electrode, it is possible to experiment only on a small area and thin thickness of the sample, and it is used only for measuring low voltages up to $1 \mathrm{kV}$. The measurement time is reduced by simultaneously measuring the volume and surface leakage currents of each insulating material using a new type of protective ring terminal electrode system for measuring the volume and surface resistance proposed in this article. Simplifying the system structure enables efficient measurements. In addition, since the resistivity can be measured at both a low voltage and high voltage, it is possible to measure the change in resistivity by the voltage magnitude.

Since the resistivity measurement is considerably influenced by various factors such as room temperature and humidity, as well as the material of the electrode, future studies will further experiment with these factors and compare them with existing measurement methods.

Author Contributions: Conceptualization, H.-G.L. and J.-G.K.; methodology, H.-G.L.; software, H.-G.L.; validation, H.-G.L.; formal analysis, H.-G.L.; investigation, H.-G.L.; resources, H.-G.L.; writing-original draft preparation, H.-G.L.; writing-review and editing, H.-G.L. and J.-G.K.; supervision, J.-G.K. All authors have read and agreed to the published version of the manuscript.

Funding: This research was supported by Korea Electric Power Corporation (Grant number: R17XA05-3).

Conflicts of Interest: The authors declare no conflict of interest.

\section{References}

1. Erika, P.; Ole, B.; Nasser, N.G.A.; Michael, K. Challenges and opportunities for a European HVDC grid. Renew. Sustain. Energy Rev. 2017, 70, 427-456.

2. Gonzalez, J.S.; Lacal-arantegui, R. A review of regulatory framework for wind energy in European Union countries: Current state and expected developments. Renew. Sustain. Energy Rev. 2016, 56, 588-602. [CrossRef]

3. Takashi, O.; Aishah, B.M.I.; Ralph, D.S. Eelectric power grid interconnections in Northeast Asia: A quantitative analysis of opportunities and challenges. Eenergy Policy 2016, 89, 311-329.

4. Zhonglei, L.; Boxue, D. Polymeric insulation for high-voltage dc extruded cables: Challenges and development directions. IEEE Electr. Insul. Mag. 2018, 34, 30-43.

5. Wu, R.; Wen, J.L.; Yu, K.; Zhao, D. A discussion of SiC prospects in next electrical grid. In Proceedings of the 2012 Asia-Pacific Power and Energy Engineering Conference, Shanghai, China, 7-29 March 2012. 
6. Muhammad, A.; Adeel, I.; Khan, N.; Muhammad, I. A gerneric scenario of a load flow study in SAARC countries super grid using high voltage alternating current and high voltage direct current. Int. J. Sci. Eng. Res. 2016, 7, 210-223.

7. Najafi, S.; Saadati, H.; Werle, P. Comparison of acoustical partial discharge signals under AC and DC stress. In Proceedings of the 2016 IEEE Electrical Insulation Conference (EIC), Montreal, QC, Canada, 19-22 June 2016; pp. 523-526.

8. Xiong, Q.; Zhu, L.; Ji, S.; Cui, Y.; Lu, W. Flashover and partial discharge characteristics of fiber of valve tower in converter station. IEEE Trans. Dielectr. Electr. Insul. 2017, 24, 1985-1991. [CrossRef]

9. ASTM D257-07. Standard Test Method for DC Resistance of Conductance of Insulating Materials; ASTM: West Conshohocken, PA, USA, 2007.

10. IEC 60093:1980. Methods of Test for Volume Resistivity and Surface Resistivity of Solid Electrical Insulating Materials; IEC: Geneva, Switzerland, 1980.

11. Yasushi, Y.; Takeyuki, K.; Masahiro, T.; Shinichi, K.; Kumi, N.; Yoshio, S. Measurement of surface and volume resistivity for alumina ceramics under vacuum condition. In Proceedings of the 2008 23rd International Symposium on Discharges and Electrical Insulation in Vacuum, Bucharest, Romania, 15-19 September 2008; pp. 35-38.

12. Frederickson, A.R.; Benson, C.E.; Bockman, J.F. Measurement of charge storage and leakage in polyimides. Ioniz. Radiat. Polym. 2003, 208, 454-460. [CrossRef]

13. Michal, L.; Ryszard, K. Changes proposed for the IEC 60093 Standard concerning measurements of the volume and surface resistivities of electrical insulating materials. IEEE Trans. Dielectr. Electr. Insul. 2003, 13, 139-145.

14. Michal, L.; Ryszard, K.; Edmund, M.; Pawel, G. Space charge influence on the results of volume resistivity measurements in solid dielectrics. In Proceedings of the XVII IMEKOWorld Congress Metrology in the 3rd Millennium, Dubrovnik, Croatia, 22-27 June 2003; pp. 837-840.

15. Eichhorn, R.M. Treeing in solid extruded electrical insulation. IEEE Trans. Eelctr. Insul. 1977, EI-12, 2-18. [CrossRef]

16. Shaw, M.T.; Shaw, S.H. Water treeing in solid dielectrics. IEEE Trans. Eelctr. Insul. 1984, EI-19, 419-452. [CrossRef]

17. Nunes, S.L.; Shaw, M.T. Water treeing in polyethylene-A review of mechanisms. IEEE Trans. Eelctr. Insul. 1984, EI-19, 419-452. [CrossRef]

18. Murata, Y.; Goshowaki, W.; Reddy, C.C.; Sekiguchi, Y.; Hishinuma, H.; Hayase, Y.; Tanaka, Y.; Takada, T. Investigation of space charge distribution and volume resistivity of XLPE/MgO nanocomposite material under DC voltage application. Int. Symp. Electr. Insul. Mater. 2008, EA-3, 502-505.

19. Chen, X.; Morshuis, P.H.F.; Smit, J.J.; Marquezin, G.; Girodet, A. Electrical properties calculation of HVDC bushing. In Proceedings of the 2010 Annual Report Conference on Electrical Insulation and Dielectic Phenomena, West Lafayette, IN, USA, 17-20 October 2010.

20. Michal, L. Issues of volume resistivity measurement of solid dielectrics and evaluation of uncertainty of results by approximate method at a confidence level of 0.95. Metrol. Meas. Syst. 2009, 16, 233-248.

21. Michal, L.; Adam, S. Effective area of thin guarded electrode in determining of permittivity and volume resistivity. IEEE Trans. Dielectr. Electr. Insul. 2009, 16, 24-31.

22. Sirvio, P.; Backfolk, K.; Maldzius, R.; Sidaravicius, J.; Montrimas, E. Dependence of paper surface and volume resistivity on electric field strength. J. Imaging Sci. Tech. 2008, 52, 30501-1-30501-8. [CrossRef]

23. Peter, P.; Randall, R.B. Using field theory to measure surface resistivity of high-resistance polymeric films. J. Appl. Polym. Sci. 2001, 82, 2856-2862.

24. Toshiyuki, S.; Makoto, A.; Yoshio, H. Noncontact surface resistivity measurement using a cylindrical surface potential detector with a corona charger. IEEE Trans. Ind. Appl. 2012, 48, 1657-1661.

25. Floran, V.; Gerhard, M.S.; Halil, S. The influence of electron-beam irradiation on the volume resistivity of polyethylene and kapton. J. Electrost. 2005, 63, 749-754.

26. Lee, S.-H.; Jang, S.-M.; Choi, J.-Y. Improvement of measuring capacity of the DC high-voltage divider for a national high-voltage standard. J. Trans. Korean Inst. Electr. Eng. 2014, 63, 1622-1625.

(C) 2020 by the authors. Licensee MDPI, Basel, Switzerland. This article is an open access article distributed under the terms and conditions of the Creative Commons Attribution (CC BY) license (http://creativecommons.org/licenses/by/4.0/). 